\title{
Exploded representation of a refracting Surface
}

\section{WH Heath* and WF Harris ${ }^{\S}$}

*, §Optometric Science Research Group, Department of Optometry, University of Johannesburg, PO Box 524, Auckland Park, 2006 South Africa

\$Department of Mathematics and Statistics, University of Johannesburg, PO Box 524, Auckland

Park, 2006 South Africa

*<optom2@na.rau.ac.za> \$<wfh@na.rau.ac.za>

\begin{abstract}
The concept of the exploded refracting surface is useful in the optics of contact lenses and vision underwater. The purpose of this paper is to show how to represent a refracting surface as an exploded pair of surfaces separated by a gap of zero width. The analysis is in terms of linear optics and allows for astigmatic and noncoaxial cases.
\end{abstract}

The concept of the exploded refracting surface is useful in contact lens optics ${ }^{1,2}$ and in the analysis of vision under water, the latter of these being a future goal of the authors. The exploded surface allows one to treat the submerged eye as being in contact with air and so retain its optical character. This allows for an easier comparison of the submerged eye to the eye in air.

The goal of this paper is to show, in general, how to represent a single refracting surface as a system of two juxtaposed single refracting surfaces. Explicit formulae are presented in equations 8 and 9 for the transferences of the two surfaces.

We make use of linear optics ${ }^{1-15}$ within which the transference is an important concept. We start by reviewing the transference ${ }^{9}$ and more particularly the transference of a single refracting surface to find the general divergence $\mathbf{C}$ and deviation $\boldsymbol{\pi}$ for any single refracting surface. From there we explode the single surface into two surfaces separated by a gap of zero width. The transferences for the two surfaces are found and from this explicit formulae are presented that can be used to calculate the transferences of the two surfaces in the exploded representation of a surface from the information contained in the transference of the original surface. The approach is general and allows for surfaces that may be astigmatic and non-coaxial.

The augmented ray transference ${ }^{9}$ is

$$
\mathbf{T}=\left(\begin{array}{lll}
\mathbf{A} & \mathbf{B} & \mathbf{e} \\
\mathbf{C} & \mathbf{D} & \boldsymbol{\pi} \\
\mathbf{o}^{\prime} & \mathbf{o}^{\prime} & 1
\end{array}\right)
$$

The $2 \times 2$ sub-matrices $\mathbf{A}, \mathbf{B}, \mathbf{C}$, and $\mathbf{D}$ are respectively called ${ }^{1}$ the dilation, disjugacy, divergence and the divarication. The $2 \times 1$ sub-matrices $\mathbf{e}$ and $\boldsymbol{\pi}$ are called the translation and deviation respectively and they allow for systems that contain tilted and decentred elements. These are the six fundamental properties of any optical system from which other properties can be derived. ${ }^{10} \mathbf{0}$ is the $2 \times 1$ null vector and $\mathbf{o}^{\prime}$ its transpose which together with a scalar 1 make up the bottom row.

\footnotetext{
BOptom

$\S$ PhD FAAO FRSSAf
} 
We will often simply refer to $\mathbf{T}$ as the transference and also often omit the bottom row. Dioptric power $\mathbf{F}$ is defined by $\mathbf{F}=-\mathbf{C} .{ }^{11}$

For a homogeneous gap the transference is ${ }^{9}$

$\mathrm{T}=\left(\begin{array}{ccc}\mathbf{I} & \boldsymbol{\zeta I} & \mathbf{0} \\ \mathbf{O} & \mathbf{I} & \mathbf{0}\end{array}\right)$,

where $\zeta$ is the reduced width of the gap. The bottom row is omitted to save space. $\mathbf{I}$ is the $2 \times 2$ identity matrix and $\mathbf{O}$ the $2 \times 2$ null matrix. A special case is the $5 \times 5$ identity transference where $\varsigma=0$.

Of particular relevance here is the transference of a single refracting surface ${ }^{9}$

$\mathrm{T}=\left(\begin{array}{lrr}\mathbf{I} & \mathbf{O} & \mathbf{0} \\ -\mathbf{F} & \mathbf{I} & \boldsymbol{\pi}\end{array}\right)$

where $\mathbf{F}$ is symmetric. ${ }^{11}$

We now examine $\mathbf{F}$ and $\boldsymbol{\pi}$ in particular.

Dioptric power can be represented as ${ }^{12}$

$\mathbf{C}=-\mathbf{F}=-\mathbf{K}\left(n_{2}-n_{1}\right)$

where $\mathbf{K}$ has been called the curvature matrix ${ }^{12}$ of the surface and $n_{1}$ and $n_{2}$ are the refractive indices of the media upstream and downstream of the surface respectively.

The deviation $\pi$ depends on the non-coaxiality of the surface with respect to the longitudinal axis $Z$. A surface normal to $Z$ has $\boldsymbol{\pi}=\mathbf{0}$. For $\boldsymbol{\pi} \neq \mathbf{0}$ the surface is tilted by $\mathbf{b}$ and/or decentered by $\mathbf{c}$. In general $^{13}$

$\boldsymbol{\pi}=(\mathbf{K c}+\mathbf{b})\left(n_{2}-n_{1}\right)$.

Next we explode $\mathrm{T}$ into two surfaces $\mathrm{T}_{1}$ and $\mathrm{T}_{2}$, that is, we imagine two surfaces that are separated by a gap of zero width, that have the same curvature as $\mathrm{T}$, and that also have the same amounts of tilt and decentration as T. Upstream of $T_{1}$ the medium has refractive index $n_{1}$, and downstream of $\mathrm{T}_{2}$ the medium has refractive index $n_{2}$. The medium in the gap between $T_{1}$ and $T_{2}$ has refractive index $n_{0}$. We refer to $n_{0}$ as the reference index. $n_{0}$ is arbitrary although in most cases it is likely to be 1. From this we know that $\mathrm{T}_{1}$ has power $\mathbf{F}_{1}=$ $\mathbf{K}\left(n_{0}-n_{1}\right)$ and deviation $\boldsymbol{\pi}_{1}=(\mathbf{K c}+\mathbf{b})\left(n_{0}-n_{1}\right)$. Similarly for $\mathrm{T}_{2}$ we have $\mathbf{F}_{2}=\mathbf{K}\left(n_{2}-n_{0}\right)$ and $\boldsymbol{\pi}_{2}$
$=(\mathbf{K c}+\mathbf{b})\left(n_{2}-n_{0}\right)$.

Using equation 4 we can represent $\mathbf{F}_{1}$ as

$\mathbf{F}_{1}=\mathbf{F} \frac{n_{0}-n_{1}}{n_{2}-n_{1}}$

and similarly for $\mathbf{F}_{2}$. Making use of equations 4 and 5 we find similar expressions for $\pi_{1}$ and $\boldsymbol{\pi}_{2}$. These results lead one to a transference for each surface in terms of $\mathbf{F}, \boldsymbol{\pi}$ and the respective refractive indices. By adding and subtracting $\mathbf{I}$ from each of the two transferences, and rearranging the terms, one finds

$$
\begin{aligned}
& \mathbf{T}_{1}=\boldsymbol{x}_{1} \mathbf{T}+\left(1-\boldsymbol{x}_{1}\right) \mathbf{I}, \\
& \text { and } \\
& \mathbf{T}_{2}=\boldsymbol{x}_{2} \mathbf{T}+\left(1-\boldsymbol{x}_{2}\right) \mathbf{I}
\end{aligned}
$$

with $\boldsymbol{x}_{1}=\frac{n_{0}-n_{1}}{n_{2}-n_{1}} \quad$ and $\quad \boldsymbol{x}_{2}=\frac{n_{2}-n_{0}}{n_{2}-n_{1}}$.

The transferences of two surfaces separated by a gap of zero width is $\mathbf{T}_{2} \mathbf{I} \mathbf{T}_{1}$. Therefore, the transference of the system of the two surfaces can simply be represented by $\mathbf{T}_{2} \mathbf{T}_{1}$ which recovers the transference $\mathbf{T}$.

The result above allows one to represent a single refracting surface as a system of two refracting surfaces, which are separated by gap of zero width that is filled with a medium with a refractive index defined as the reference index. Therefore, given any transference of a single refracting surface $T$, one can design a system of two juxtaposed refracting surfaces $T_{1}$ and $T_{2}$, that will yield the same transference.

\section{Acknowledgements}

This work is supported by the National Research Foundation of South Africa under grant number 2053699 to Professor WF Harris and forms part of the work being done by WH Heath for the degree DPhil under the guidance of Professor WF Harris.

This work forms part of a poster ${ }^{16}$ presented at the Annual Meeting of the American Academy of Optometry in Tampa, Florida (USA) on the $10^{\text {th }}$ of December 2004. 


\section{References}

1. Harris WF. Image size magnification and power and dilation factors for optical instruments in general. Ophthal Physiol Opt 2003 23 251-261.

2. Harris WF. Magnification, blur, and ray state at the retina for the general eye with and without a general optical instrument in front of it. 1. Distant objects. Optom Vis Sci 200178 888-900.

3. Long WF. A matrix formalism for decentration problems. Am J Optom Physiol Opt 197653 27-33.

4. Keating MP. An easier method to obtain the sphere, cylinder and axis from an off-axis dioptric matrix. Am J Optom Physiol Opt 198057 734-7.

5. Keating MP. A system matrix for astigmatic optical systems: I. Introduction and dioptric power relations. Am J Optom Physiol Opt 198158 810-9.

6. Keating MP. A system matrix for astigmatic optical systems: II. Corrected systems including an astigmatic eye. Am J Optom Physiol Opt 198158 919-29.

7. Keating MP. Lens effectivity in terms of dioptric power matrices. Am J Optom Physiol Opt 198158 1154-60.

8. Keating MP. Advantages of a block matrix formulation for an astigmatic system. $A m J$ Optom Physiol Opt 198259 851-7.

9. Harris WF. Paraxial ray tracing through noncoaxial astigmatic optical systems, and a $5 \times 5$ augmented system matrix. Optom Vis Sci 199471 282-285.

10. Harris WF. A unified paraxial approach to astigmatic optics. Optom Vis Sci 199976 480-499.

11. Harris WF. Dioptric power: its nature and its representation in three- and four- dimensional space. Optom Vis Sci 199774 349366.

12. Harris WF. The second fundamental form of a surface and its relation to the dioptric power matrix, sagitta and lens thickness. Ophthal Physiol Opt 19899 415-9.

13. Harris WF. Astigmatic optical systems with separated and prismatic or noncoaxial elements: System matrices and system vectors. Optom Vis Sci 199370 545-551.

14. Fick HH. Fortschrittliche rechnungsarten in der augenoptik. Folge 11. Der Augenoptiker 197212 60-63.

15. Fick HH. Fortschrittliche rechnungsarten in der augenoptik. Folge 12. Der Augenoptiker 19732 45-49.

16. Heath WH, Harris WF. The linear optics of the general submerged eye. Optom Vis Sci 200481 (12 S) 163. 